\title{
Desarrollo de un sistema de gestión para la seguridad de la información basado en metodología de identificación y análisis de riesgo en bibliotecas universitarias
}

\author{
Erick Guerra, Harold Neira, Jorge L. Díaz y Janns Patiño \\ Dpto. de Ciencias de la computación y electrónica, Universidad de la costa, Barranquilla -Colombia \\ (Correo-e: eguerra4@cuc.edu.co; hneira@cuc.edu.co; Jdiaz5@cuc.edu.co; Jpatino8@cuc.edu.co)
}

Recibido May. 4, 2021; Aceptado Jun. 30, 2021; Versión final Jul. 26, 2021, Publicado Oct. 2021

\begin{abstract}
Resumen
La presente investigación tiene como objeto de estudio aplicar un sistema de gestión de la información basado en la metodología de identificación y análisis de riesgos para los procesos de bibliotecas universitarias. Se adapta la norma ISO/IEC 27001:2013 aplicando la metodología MARGERIT en una biblioteca universitaria. Los resultados obtenidos de los cálculos de riesgos intrínseco y efectivo demuestran la presencia de salvaguardas y la evaluación de los impactos. Se establece el porcentaje de afectación en cada riesgo por proceso de calidad, se identifica la medida correctiva, y se incorporan formatos de registros. Se concluye que la incorporación de los formatos propuestos para desarrollar el control y auditorías a los indicadores de calidad permite la optimización del sistema de gestión de la seguridad de la información (SGSI) para los procesos de la biblioteca universitaria.
\end{abstract}

Palabras clave: gestión; seguridad; informática; metodología; identificación; análisis; riesgo

\section{Development of an information security management system based on analysis methodology and risk identification in university libraries}

\begin{abstract}
The primary objective of this research study is to apply an information management system based on analysis methodology and risk identification for university library processes. The ISO/IEC 27001:2013 standard is adapted and applied by using the MARGERIT methodology to assess a university library. The results obtained from the intrinsic and effective risk calculations show the presence of safeguards and the evaluation of impacts. The percentage of influence in each risk by quality process is established, corrective measures are identified, and record formats are incorporated. It is concluded that incorporating the proposed formats to develop quality indicator controls and audits allow optimizing the information security management system (SGSI, in Spanish) of university library processes.
\end{abstract}




\section{INTRODUCCIÓN}

En la actualidad, las organizaciones sin importar su clasificación poseen diferentes tipos de activos tales como infraestructura o planta física, mobiliario, equipos de computación, sistemas de redes y comunicaciones, maquinarias, dinero en efectivo, vehículos entre otras. Sin embargo, el activo más importante que algunas veces pasa desapercibido por el gobierno corporativo y de la administración de las organizaciones es la información (Zhao et al, 2020; Zhang, 2021). Del mismo modo, hoy en día se reconoce que algunos de los problemas más importantes que atraviesan dichas organizaciones se relacionan con el tráfico, espionaje y robo de información (Haghighat y Li, 2021).

De este modo, se debe reconocer el elevado valor que posee hoy en día la información para cualquier organización, pues esta se posiciona como el elemento clave de la actividad de cualquier empresa en la actualidad y mucho más cuando se considera que la sociedad actual le da un elevado rol a la gestión y circulación de la información, apoyándose en las TIC (Lay et al., 2019; Sánchez-Otero et al., 2019); las cuales han generado un cambio significativo en el ámbito organizacional hacia la eficiencia y transparencia en dicho proceso de gestión (Villarreal et al., 2019); afectando así a muchas organizaciones que realizan sus procesos por medio del internet, estando expuestas a vulneraciones de la misma (Chang et al., 2020). Es así, como resulta fundamental que dicho sistema sea eficiente y se maneje bajo los mayores estándares de seguridad que permitan a las empresas gestionar su información de manera eficaz y confiando en que todo esa data se encuentra sumamente protegida contra cualquier tipo de atentado, que podría afectar de manera significativa no solo la información existente en esta, sino la misma confianza que la organización representa en el mercado y que le respalda para poder seguir estando dentro de los estándares competitivos de la actualidad (Liu et al., 2021).

De acuerdo con Bauer y Bernroider (2017) la política de seguridad de la información implementada por la organización para proteger su información puede ser uno de los temas que pueden generar controversia, la razón es que, a pesar de la existencia de esta información, la seguridad de la información es violada por factores humanos. Los diferentes roles que desempeñan las personas, como usuarios finales, administradores de equipos de seguridad, administradores de información, administradores de políticas de seguridad, atacantes de sistemas de información que tendrán diferentes efectos y consecuencias para cada situación. Como consecuencia, la seguridad de la información no es sólo cuestión de tener nombres de usuario y contraseñas, sino que requiere normativas y diversas estrategias de privacidad y protección de datos que impongan obligaciones a la organización (Safa et al., 2016). También debe ser integral y adecuada para empresas que no tengan problemas. Además de lo anterior: seguridad del personal, control de acceso de usuarios, también debe incluir: seguro aspecto redes y regulaciones (Susanto y bin Muahaya, 2010).

Las anteriores obligaciones que se ejercen bajo la seguridad de la información pueden ser solventadas con la ayuda de un Sistema de gestión de la seguridad de la Información (SGSI) activos de información, minimizando posibles riesgos que atenten contra la misma (Qui et al., 2020). Los sistema de gestión de la seguridad de la información (SGSI) están dirigidos a entender las propuestas de gestión de seguridad de la información, al respecto las bibliotecas manejan activos y procesos a fines a cualquier tipo de negocio o empresa, lo que permite establecer que los modelos de gestión de seguridad no son aplicables exclusivamente a las bibliotecas si no cualquier tipo de negocio en general, estos modelos están basado en el uso del conjunto de la norma 27001 del organismo internacional de estandarización (ISO por sus siglas en inglés), también llamada norma ISO 27001 (Jamandilović y Stojanović, 2018).

Este tipo de sistema de gestión de la seguridad de la información (SGSI) esta direccionado hacia las metodologías de análisis de riesgo, como lo son las metodologías OCTAVE, NIST SP 800:30, MARGERIT, lo que permite garantizar la implementación de SGSI, previa adaptaciones y estandarización de los procesos que describen a una empresa o para el caso de estudio los procesos de la Biblioteca (Tigănoaia, 2015). Al respecto, la norma ISO/IEC 27001 (2013) ha sido desarrollada con el fin de servir como el modelo para el establecimiento, implementación, seguimiento, y mejora de un SGSI en cualquier tipo de organización.

La implementación de adaptaciones de la metodología a procesos específicos establece mecanismos como indicadores, que tiene como objeto evaluar y medir el nivel de cumplimiento de cada procedimiento y tareas, resguardar el proceso macro y la seguridad de la información relacionada. Al respecto de seguridad las bibliotecas manejan información confidencial sobre sus usuarios tales como: Gestión de multas, contraseñas, material bibliográfico, cuentas de usuarios, conformando los activos más relevantes dentro de la organización, al igual que el inventario de todos los activos que conforman la biblioteca, todos estos datos requieren de niveles de seguridad, por ende, se deben salvaguardar de una manera eficiente desde su origen.

Tomando en cuenta lo anterior, la presente investigación se desarrolla con el objeto de aplicar un Sistema de gestión de la información basado en la metodología de identificación y análisis de riesgos para los procesos 
de bibliotecas universitarias. Para lograr dicho objetivo, se selecciona una biblioteca de una institución universitaria, la cual se selecciona por no contar con procesos automatizados referentes a la seguridad y la estandarización, careciendo de un sistema que gestione la seguridad de la información. Es importante destacar, que la gestión de la calidad y la estandarización de los procesos no son controlados a través de indicadores y normas, generando robo, pérdida y falta de disponibilidad de la información, razón por la cual se requiere el desarrollo de un Sistema de Gestión de Seguridad de la Información, sustentado en la metodología de análisis de riesgos, el cual debe ser compatible con la norma ISO/IEC 27001 (2013), lo cual permitirá garantizar los principios de seguridad de la información de los usuarios. Ciertamente, esta realidad tiene un gran efecto sobre las instituciones de educación superior; las cuales en aras de formar parte en los procesos de alta calidad buscan en la innovación tecnológica una herramienta para lograr el posicionamiento y reconocimiento en el fomento de un ambiente direccionado a la alta calidad educativa, apoyada efectivamente en las nuevas tecnologías como pilar de dicho proceso (Hernández et al., 2017).

\section{METODOLOGÍA}

Para el desarrollo del presente estudio se desarrolla una metodología de tipo aplicada que según lo estipula Chávez (2007, p. 134) esta está: "Dirigida a la aplicación inmediata mediante acciones concretas para enfrentar el problema. Se dirige a la acción inminente y no al desarrollo por la teoría y sus resultados, mediante actividades precisas para enfrentar el problema". A partir de esto, la investigación es dividida en dos fases: la primera direccionada al proceso de recolección documental de la información y la construcción del sistema de gestión para la seguridad de la información basado en metodología de identificación y análisis de riesgo en bibliotecas universitarias, y el segundo enfocado al proceso de implementación del mismo sistema dentro de una biblioteca de una institución universitaria localizada en la ciudad de Barranquilla, Colombia. De este modo, partiendo de la revisión documental y otras fuentes de información, se estableció la conceptualización de las nociones de seguridad de la información y análisis de riesgos, sus aspectos, importancia, enfoques y características, así como la caracterización de metodologías basada en normas ISO. El marco referencial orientado a los antecedentes del sistema de la gestión de la información permite definir y aclarar lo que se pretende conseguir y cómo se debe alcanzar, de acuerdo con un procedimiento establecido.

La metodología para la gestión de los procesos de la biblioteca se plasma en un documento donde se concreta las directrices, indicadores y elementos que van a orientar la gestión de la calidad de los procesos de la Biblioteca universitaria seleccionada para la aplicación de la metodología propuesta. A su vez, el desarrollo de un SGSI basado en la metodología de identificación y análisis de riesgos, para los procesos de la biblioteca seleccionada, está orientado a sistematizar, organizar, priorizar, estructurar: procesos, procedimientos y funciones que se efectúa en la Biblioteca, como unidad de estudio a través de la adaptación de la metodología y norma internacional ISO/IEC 27001 (2013). Con respecto a la identificación y análisis de riesgo, de los procesos estandarizados por la norma, será necesario adaptar e integrar la metodología de análisis de riesgo MAGERIT, la cual se caracteriza por estudiar las alarmas mediante un proceso sistemático, para estimar la magnitud de lo que está expuesta una organización teniendo en cuenta los conflictos derivados del uso de las TICS. Este proceso requiere de la puesta en marcha de 10 fases mostradas en la figura 1:

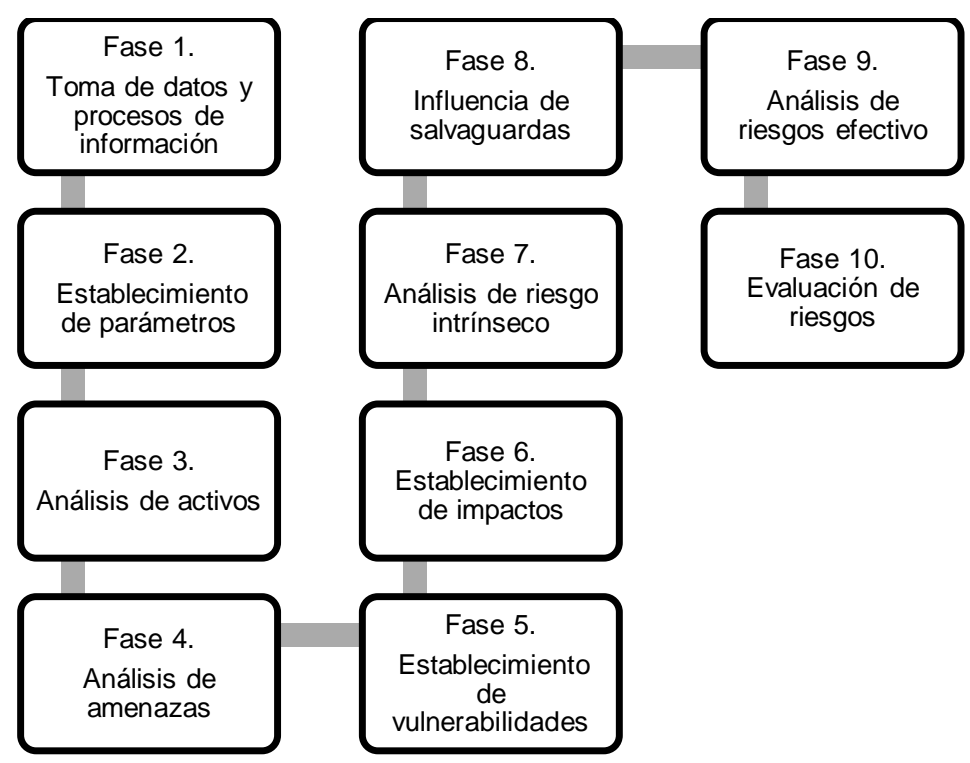

Fig. 1: Fases de la metodología 


\section{RESULTADOS Y DISCUSIÓN}

Para el desarrollo del SGSI, luego de la revisión de los métodos establecidos por Jamandilović y Stojanović (2018), se establecen como proceso a seguir la implementación de la metodología MAGERIT para la gestión de los riesgos, conformada por diez fases: alcance del SGSI, identificación de los parámetros, análisis de activos, análisis de amenazas, establecimiento de vulnerabilidades, establecimiento de impactos, análisis de riesgo intrínseco, influencia de salvaguardas, análisis de riesgos efectivo, y evaluación de riesgos:

Fase 1. En esta fase se establece el alcance del SGSI para el manejo de riesgos de la biblioteca de la universidad seleccionada, a través de la identificación de los procesos bases. Para el SGSI se utilizó como fuente el manual de calidad de dicha, lo cual permitió identificar y analizar siete (7) procesos bases de la biblioteca que se encuentran establecidos en dicho manual de calidad de la universidad que serán objeto de estudio del sistema del SGSI. Estos siete procesos son: el adquirir material, incorporación a colección, circular material, formar usuarios, gestión interbibliotecaria, uso de recursos electrónicos y sala de consulta especializada. Según la norma ISO, y en acuerdo con lo expuesto por Jamandilović y Stojanović (2018), cada proceso presenta sus entradas, funciones y salidas, con el objeto de asegurar la calidad, de acuerdo con el manual de calidad de la universidad.

Fase 2. Identificar los parámetros que se utilizarán durante todo el proceso de análisis de riesgos: Para identificar y definir los parámetros, que serán base para el proceso de análisis de riesgos, se hace necesario determinar el valor de los activos y la vulnerabilidad del proceso, esto se logró por medio de las actividades de observación directa y revisión documental. Para determinar el valor de los activos, se establece el inventario de la biblioteca en cuanto: activos como material bibliográfico, equipo de mobiliario y tecnología necesario para el funcionamiento del proceso de biblioteca. Para efectos de cálculos necesarios en la presente investigación, se establecen como costos promedios. De acuerdo con los valores establecidos en el SGC, la frecuencia de ocurrencia de los riesgos no se encuentra definida actualmente, de acuerdo con los procedimientos que se desarrollan dentro de la biblioteca, sin embargo, se establecen los niveles de probabilidad y de impacto de los riesgos en el SGC. Se procedió a estudiar las alarmas asociadas a los procesos de calidad, de acuerdo con el Sistema de la Gestión de la Calidad, se establece la clasificación en ocho (8) tipos de peligros mostrados a continuación en la tabla 1:

Tabla 1: Clasificación de Riesgos de la biblioteca

\begin{tabular}{|c|c|}
\hline Tipo de riesgo & Riesgo \\
\hline $\begin{array}{l}\text { Riesgos estratégicos } \\
(\mathrm{Re})\end{array}$ & $\begin{array}{l}\text { Está asociado con métodos de gestión de identidad. La gestión de riesgos estratégicos se } \\
\text { centra en cuestiones globales relacionadas con el cumplimiento de las tareas y los objetivos } \\
\text { estratégicos, la definición clara de políticas, el diseño y conceptualización de entidades de la } \\
\text { alta dirección. }\end{array}$ \\
\hline Riesgos operativos (Ro) & $\begin{array}{l}\text { Incluye riesgos relacionados con las partes operativas y técnicas de la entidad, a su vez que } \\
\text { la ineficiencia causada por la separación de sistemas de información, definiciones de } \\
\text { procesos, estructura de la entidad y dependencias, corrupción y oportunidades de } \\
\text { incumplimiento de compromisos institucionales. }\end{array}$ \\
\hline Riesgos financieros (Rf) & $\begin{array}{l}\text { Están relacionados con la gestión de los recursos físicos, incluida la ejecución del } \\
\text { presupuesto, la preparación de estados financieros, el pago, la gestión de exceso de } \\
\text { inventario y la gestión de cada activo físico. Del mismo modo, la eficiencia y transparencia de } \\
\text { la gestión de recursos, y su interacción con otras áreas, dependerá en gran medida del éxito } \\
\text { o fracaso de cualquier entidad. }\end{array}$ \\
\hline $\begin{array}{l}\text { Riesgos de cumplimiento } \\
\text { (Rc) }\end{array}$ & $\begin{array}{l}\text { Están relacionados con la capacidad de la entidad para cumplir con las leyes, los contratos, } \\
\text { los requisitos de moral pública y el compromiso general con la comunidad. }\end{array}$ \\
\hline $\begin{array}{l}\text { Riesgo de tecnología } \\
\text { (Rt) }\end{array}$ & $\begin{array}{l}\text { Están asociados con las capacidades de la entidad, por lo que la tecnología disponible puede } \\
\text { satisfacer sus necesidades actuales y futuras, del mismo modo apoyar la realización de } \\
\text { tareas. }\end{array}$ \\
\hline $\begin{array}{l}\text { Riesgo de talento } \\
\text { humano (Rth) }\end{array}$ & $\begin{array}{l}\text { Se asocia con la capacidad de la entidad para cumplir con las necesidades y brindar soporte } \\
\text { a sus empleados directos e indirectos. Como incumplimiento en la legalización de contratos, } \\
\text { incumplimiento en las respuestas a solicitudes y requerimientos, inoportunidad en la } \\
\text { liquidación de nómina, entre otros. }\end{array}$ \\
\hline Riesgo legal (RI) & $\begin{array}{l}\text { Se asocia al desconocimiento del marco legal al que se sujeta la Universidad, ejemplos: } \\
\text { respuestas a derechos de petición, tutelas, entre otros. }\end{array}$ \\
\hline Riesgo ambiental (Ra) & $\begin{array}{l}\text { Probabilidad que se produzca, genere un daño o catástrofe, emergencia por modificación o } \\
\text { alteración del Sistema de Gestión Ambiental }\end{array}$ \\
\hline
\end{tabular}

Fase 3. Análisis de activos: Luego de identificar los procesos de calidad, el valor de los activos, riesgos, vulnerabilidad y frecuencia de ocurrencia se hace necesario identificar los inventarios de recursos necesarios para los procesos de la biblioteca seleccionada para el estudio, los cuales están asociados a: material bibliográfico, tecnológico, inmobiliario y sistema. 
Fase 4. Análisis de amenazas: De acuerdo con la norma ISO 27001, la consecuencia de las amenazas es un incidente que modifica el estado de seguridad de los activos, por tal razón se deben clasificar y codificar las amenazas en cuatro (4) rubros importantes entre ellas: no humanas (A), humanas (AH), humanas intencionales que necesitan presencia física (Al), humana intencional que proceden de un origen remoto (AR). Entre ellas se pueden detallar de acuerdo con el rubro.

Fase 5. Establecimiento de vulnerabilidades: para ello debe quedar claro que, sin vulnerabilidad, las amenazas no dañarán los activos, las lagunas en sí mismas no causarán daños, pero siempre serán causadas por amenazas. La vulnerabilidad intrínseca puede descomponerse en análisis detallados, que se encuentran en varios bloques de atributos.

Fase 6. Establecimiento de impactos: Identificadas las amenazas y vulnerabilidades de la biblioteca; se representa la combinación de las amenazas y vulnerabilidades, previamente clasificadas y codificadas a través de niveles en cinco categorías, entre ellos: Muy alto 100\% (rojo), Alto 75 - $99 \%$ (naranja), Medio 50 - $75 \%$ (amarillo), Bajo $20-50 \%$ (verde), Muy bajo 5-20\% (azul). La utilidad de la gráfica se deriva, en el reconocimiento del impacto, que pueden producir las vulnerabilidades sobre las amenazas, generando como consecuencia un riesgo; Es importante destacar, que reducir el riesgo depende de la gestión, acción y control efectivo que hagan los encargados y todo el personal asociado a los procesos de la biblioteca. De acuerdo con los análisis anteriores, se lograron identificar los niveles, para calificar el impacto de las vulnerabilidades, amenazas sobre los riesgos.

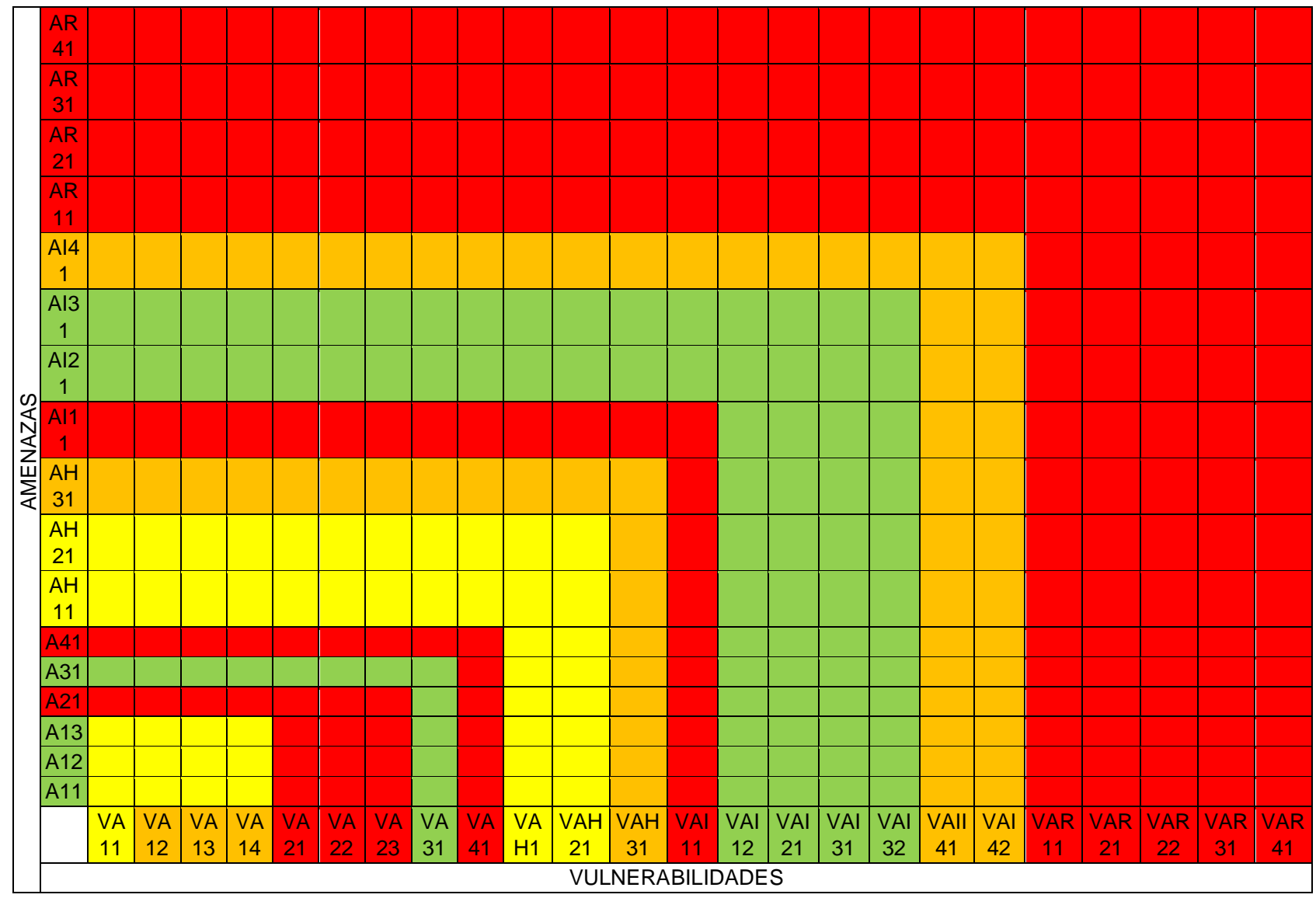

Fig. 2: Impacto de amenazas y vulnerabilidades.

Fase 7. Análisis de riesgo intrínseco: Para generar los cálculos, relacionados con la estimación del riego intrínseco, se tomará como base la sumatoria total de los activos por rubro, así como la escala de niveles de impacto y vulnerabilidad. Así mismo, se hace necesario definir escala de parámetros para las amenazas y vulnerabilidades identificadas, al respecto de los costos de los activos se le asignó la categoría de valor: muy alto, alto, medio y bajo, debido a que la relación se establece en función de los costos. Los resultados obtenidos del cálculo, relacionados con riesgo intrínseco, reflejan que las amenazas pueden afectar un $98,49 \%$, lo que representa el porcentaje del valor intrínseco en cualquier de los procesos de calidad, de la Biblioteca universitaria seleccionada para la puesta en marcha de la propuesta: 
Tabla 2: Análisis de riesgo intrínseco de la Biblioteca

\begin{tabular}{|c|c|c|c|c|}
\hline Amenaza: No Humanas & Vulnerabilia & & & \\
\hline A11: Pisos mojados o húmedos & PF & & & \\
\hline A12: Incendios accidentales & (Poco Frecuente) & 0,03 & A & 90 \\
\hline A13: Explosión por acumulación de gases & & & & \\
\hline & PF & & & \\
\hline A31:Temblor, sismos & (Poco Frecuente) & 0,03 & $\mathrm{~B}$ & 10 \\
\hline A41: Suspensión del servicio eléctrico & $\mathrm{FN}$ & & & \\
\hline & (Frecuente Normal) & 0,05 & $\mathrm{M}$ & 50 \\
\hline Amenaza: Humanas & & & & \\
\hline AH11: Desconocimiento en el uso de & & & & \\
\hline equipos de computación & MF & 0,071 & A & 90 \\
\hline & (Muy Frecuente) & & & \\
\hline AH21:Problemas en la Transcripción de & MF & & & \\
\hline información & (Muy Frecuente) & 0,071 & M & 50 \\
\hline AH31: Falta de supervisión & $\mathrm{F}$ & 0,016 & A & 90 \\
\hline & (Frecuente) & & & \\
\hline Amenaza: Humanas Intencionales & & & & \\
\hline Al11:Biblioteca cerrada & PF & & & \\
\hline & (Poco Frecuente) & 0,03 & $\mathrm{~B}$ & 10 \\
\hline Al21: Información mal cargada en el & MF & & & \\
\hline sistema & (Muy Frecuente) & 0,071 & $\mathrm{M}$ & 50 \\
\hline Al31:Ausencia del personal & MF & & & \\
\hline & (Muy Frecuente) & 0,071 & $\mathrm{M}$ & 50 \\
\hline Al41: Disponibilidad de información & $\mathrm{F}$ & 0,016 & $\mathrm{M}$ & 50 \\
\hline & (Frecuente) & & & \\
\hline $\begin{array}{l}\text { Amenaza: Humanas Intencionales de origen } \\
\text { remoto }\end{array}$ & & & & \\
\hline AR11: Acceso no autorizado & $\mathrm{F}$ & 0,016 & $A$ & 90 \\
\hline & (Frecuente) & & & \\
\hline AR21: Acceso con violación de permisos & $\mathrm{F}$ & 0,016 & A & 90 \\
\hline & (Frecuente) & & & \\
\hline AR31: Acceso con identidad falsa & $\mathrm{F}$ & 0,016 & A & 90 \\
\hline & (Frecuente) & & & \\
\hline $\begin{array}{l}\text { AR41: Dirección de origen bloqueada, no } \\
\text { autorizada. }\end{array}$ & $\mathrm{F}$ & 0,016 & A & 90 \\
\hline & (Frecuente) & & & \\
\hline
\end{tabular}

Fase 8. Influencia de salvaguardas: Las salvaguardas se construyen en función de las amenazas, que pueden generar riesgos, basados en la Análisis de riesgo intrínseco, por cada amenaza se elabora la salvaguarda, indicando a través de los indicadores vulnerabilidad e impacto.

Fase 9. Análisis de riesgos efectivo: Analizando los resultados, el riesgo efectivo sería de $74,82 \%$ en el caso de materializarse las amenazas, sin embargo, con la presencia de las salvaguardas esto se reduciría al $25,18 \%$

Fase 10. Evaluación de riesgos: Luego de haber determinado los riesgos existentes en la Biblioteca, así como las medidas adecuadas para disminuirlos, se establecen alternativas para afrontar los riesgos entre ellas: eliminar, transferir, asumir o mitigar el riesgo. La identificación de los riesgos, controles y medidas de mitigación ha permitido establecer los responsables o entidades a cargo de la aparición de cada uno de ellos, logrando así asociar estrategias que permiten tal como lo establece la norma, disminuir, hasta eliminar el riesgo.

Finalizada la implementación del SGSI en la biblioteca universitaria seleccionada, se logró establecer que existen ausencia de información en la documentación que respalda al Sistema de la gestión de la Calidad, motivo por el cual se propone la inclusión de dos (2) formatos sus formularios de clasificación de los riesgos, probabilidad, tabla de análisis de impacto, los cuales están asociados al registro de amenazas y vulnerabilidades por proceso, así como el impacto que puede tener, así mismo se sugiere; incorporar los cálculos de los riesgos intrínsecos y efectivos, que permitan suministrar información a la alta gerencia, con el objeto de apoyar a la toma de decisiones, de igual forma se sugiere incorporar el formato de análisis de riesgo. De este modo, se pueden contrastar los hallazgos de este estudio con los comentados por parte de Valencia y Orozco (2017) quienes a su vez también proponen un sistema de gestión de la seguridad de la información en las organizaciones basándose en normativas de índole internacional como lo son la ISO/IEC 27001, la ISO/ IEC 27002, ISO/IEC 27005 y la ISO/IEC 27003. No obstante, en este caso los autores realizan una propuesta metodológica distribuida en diez pasos, los cuales no fueron empleados en una prueba piloto, limitando el alcance de dicho estudio citado. 
Tabla 3: Análisis salvaguardas de la Biblioteca

\begin{tabular}{|c|c|c|c|c|c|}
\hline \multirow{2}{*}{$\begin{array}{l}\text { Amenaza: No Humanas } \\
\text { A11: Pisos mojados o húmedos } \\
\text { A12: Incendios accidentales A13: } \\
\text { Explosión por acumulación de } \\
\text { gases }\end{array}$} & \multirow{2}{*}{$\begin{array}{l}\text { Salvaguarda } \\
\text { S1: Proponer intercambio de } \\
\text { espacios. } \\
\text { S2: Realizar rediseño de planes de } \\
\text { Higiene y seguridad (humedad, } \\
\text { accidentes). }\end{array}$} & \multicolumn{2}{|c|}{$\begin{array}{c}\text { Disminución } \\
\text { Vulnerabilidad } \\
\%\end{array}$} & \multicolumn{2}{|c|}{$\begin{array}{l}\text { Disminución } \\
\text { Impacto \% }\end{array}$} \\
\hline & & $\mathrm{M}$ & 60 & $A$ & 90 \\
\hline A31: Temblor, sismos & S3: Validar plan de evacuación & $\mathrm{M}$ & 60 & $\mathrm{M}$ & 60 \\
\hline $\begin{array}{l}\text { A41: Suspensión del servicio } \\
\text { eléctrico }\end{array}$ & $\begin{array}{l}\text { S4: Instalación de sistema eléctrico e } \\
\text { independiente a través de pantas } \\
\text { industriales }\end{array}$ & $\mathrm{M}$ & 60 & A & 90 \\
\hline \multicolumn{6}{|l|}{ Amenaza: Humanas } \\
\hline $\begin{array}{l}\text { AH11: Desconocimiento en el uso } \\
\text { de equipos de computación }\end{array}$ & $\begin{array}{l}\text { S5: Capacitación personal interno y } \\
\text { externo. }\end{array}$ & $\mathrm{M}$ & 60 & A & 90 \\
\hline $\begin{array}{l}\text { AH21: Problemas en la } \\
\text { Transcripción de información }\end{array}$ & $\begin{array}{l}\text { S6: Entrenamiento con relación al } \\
\text { Sistema }\end{array}$ & $\mathrm{M}$ & 60 & A & 90 \\
\hline AH31: Falta de supervisión & $\begin{array}{l}\text { S7: Revisión de perfiles de cargo y } \\
\text { metas de supervisión. }\end{array}$ & $\mathrm{M}$ & 60 & A & 90 \\
\hline \multicolumn{6}{|l|}{$\begin{array}{l}\text { Amenaza: Humanas } \\
\text { Intencionales }\end{array}$} \\
\hline Al11: Biblioteca cerrada & $\begin{array}{l}\text { S8: Establecer horario de } \\
\text { atención }\end{array}$ & A & 90 & $\mathrm{M}$ & 60 \\
\hline $\begin{array}{l}\text { Al21: Información mal cargada en } \\
\text { el sistema }\end{array}$ & S9: Depurar base de datos & A & 90 & A & 90 \\
\hline AI31: Ausencia del personal & $\begin{array}{l}\text { S10: Supervisar el personal de } \\
\text { biblioteca }\end{array}$ & A & 60 & $\mathrm{M}$ & 60 \\
\hline $\begin{array}{l}\text { Al41: Disponibilidad de } \\
\text { información }\end{array}$ & $\begin{array}{l}\text { S11: Validar funcionamiento del } \\
\text { Sistema }\end{array}$ & A & 90 & $A$ & 90 \\
\hline \multicolumn{6}{|l|}{$\begin{array}{l}\text { Amenaza: Humanas } \\
\text { Intencionales de origen remoto }\end{array}$} \\
\hline $\begin{array}{l}\text { AR11: Acceso no } \\
\text { autorizado }\end{array}$ & \multirow{4}{*}{$\begin{array}{l}\text { S12: Establecer niveles de } \\
\text { seguridad, actualización de licencias } \\
\text { y sistemas }\end{array}$} & A & 90 & A & 90 \\
\hline $\begin{array}{l}\text { AR21: Acceso con } \\
\text { violación de permisos }\end{array}$ & & $A$ & 90 & A & 90 \\
\hline $\begin{array}{l}\text { AR31: Acceso con } \\
\text { identidad falsa }\end{array}$ & & A & 90 & A & 90 \\
\hline $\begin{array}{l}\text { AR41: Dirección de origen } \\
\text { bloqueada, no } \\
\text { autorizada. }\end{array}$ & & $A$ & 90 & $A$ & 90 \\
\hline
\end{tabular}

Por otro lado, Bravo (2018) quien buscó desarrollar un sistema de gestión de la seguridad de la información para bibliotecas basado en una metodología mejorada de análisis de riesgos optimizado para los activos y procesos que sean compatibles con la norma ISO/IEC 27001:2013 dentro de una biblioteca; donde se destaca que la misma autora encuentra que en dicha organización se le da una importancia muy baja a los controles establecidos por la normatividad, lo cual trae como resultado que la gran mayoría de los controles se encuentren en un 10\% de su puesta en marcha, estando la gran mayoría en las etapas iniciales. Dicha realidad también es encontrada por parte de Guamán (2019) quien evaluó la seguridad de la información dentro de una institución educativa, en este caso en el sistema de evaluación docente, encontrando que con certeza que las organizaciones actualmente no cuentan con los sistemas, políticas y mecanismos adecuados para asegurar dicha seguridad.

Es importante reconocer, tal como menciona Tigănoaia (2015) y Liu et al. (2021), el gran valor que posee la seguridad dentro de cualquier plataforma tecnológica, pues ello permite generar seguridad en los usuarios sobre dichas plataformas y que toda la información depositada dentro de esta se encuentra protegida bajo todos los lineamientos relacionados a la calidad dentro del ámbito organizacional, recordando ciertamente como la información hoy en día es uno de los activos más importantes que cualquier empresa puede poseer. Tomando en cuenta lo mencionado, es fundamental citar a Akinola (2020) y Khan y Ayesha (2021) cuando explican la gran importancia que tienen las bibliotecas como elemento clave en el desarrollo del conocimiento dentro de las instituciones de educación superior; especialmente frente al importante salto tecnológico que ha creado un nuevo ambiente digital donde el saber alcanza cada vez nuevos horizontes, permitiéndole ampliar de manera importante el impacto de esta. No es de extrañar entonces que el fortalecimiento de los entornos virtuales de aprendizaje vaya a la par del fortalecimiento de los sistemas de seguridad de estos, pues esto es lo que garantiza la preservación de los estándares de calidad, tal como plantea Donaldson (2020). 
Tabla 4: Evaluación de riegos

\begin{tabular}{|c|c|c|c|c|c|c|c|c|c|}
\hline & & & Dlasific & caciór & on del & riesg & & & \\
\hline $\begin{array}{l}\text { Descripción Del } \\
\text { Riesgo }\end{array}$ & Control Actual & $R e$ & Ro R & $R f \mid R C$ & c Rt & Rth & $R / R a$ & Medidas De Mitigación & Estrategia \\
\hline $\begin{array}{l}\text { Incumplimiento en la } \\
\text { entrega del material } \\
\text { según la planificación }\end{array}$ & $\begin{array}{l}\text { Planificación espaciada, confirmación } \\
\text { por correo de la actividad, notificación } 2 \\
\text { horas antes en caso de posible } \\
\text { incumplimiento }\end{array}$ & & $X$ & $x$ & & & & $\begin{array}{l}\text { Asignación de } \\
\text { responsables del } \\
\text { seguimiento del } \\
\text { servicio por turno, } \\
\text { medición a través de la } \\
\text { encuesta de } \\
\text { satisfacción. } \\
\end{array}$ & $\begin{array}{l}\text { Diseño de una oferta } \\
\text { de servicios que } \\
\text { satisfaga las } \\
\text { necesidades de la } \\
\text { docencia y la } \\
\text { investigación. }\end{array}$ \\
\hline $\begin{array}{l}\text { No encontrar planta } \\
\text { física disponible para } \\
\text { la capacitación }\end{array}$ & $\begin{array}{l}\text { Envío de avances sobre la formación de } \\
\text { usuarios de los programas. (Estadística } \\
\text { mensual entregada a Vice Académica) }\end{array}$ & & $X$ & & & & & $\begin{array}{l}\text { Gestionar un espacio } \\
\text { físico para } \\
\text { capacitaciones de } \\
\text { Biblioteca. En largo } \\
\text { plazo, proyecto edificio } \\
\text { de Biblioteca. }\end{array}$ & \multirow{3}{*}{$\begin{array}{l}\text { Incremento de } \\
\text { consulta y consumo } \\
\text { de los servicios de } \\
\text { biblioteca, mediante } \\
\text { el fortalecimiento del } \\
\text { programa de } \\
\text { competencias } \\
\text { informacionales a } \\
\text { estudiantes y } \\
\text { profesores. } \\
\text { Procedimiento } \\
\text { formación de } \\
\text { usuarios }\end{array}$} \\
\hline $\begin{array}{l}\text { Estudiantes que no } \\
\text { asisten a las } \\
\text { capacitaciones }\end{array}$ & $\begin{array}{l}\text { Contactar vía email para ofrecer la } \\
\text { capacitación }\end{array}$ & & X & & & & & $\begin{array}{l}\text { Mantener controles } \\
\text { aplicados }\end{array}$ & \\
\hline $\begin{array}{l}\text { Incumplimiento en las } \\
\text { metas de } \\
\text { capacitaciones }\end{array}$ & $\begin{array}{l}\text { Información constante sobre el } \\
\text { programa de formación de usuarios de } \\
\text { la biblioteca }\end{array}$ & & $X$ & & & & & $\begin{array}{l}\text { Mantener controles } \\
\text { aplicados }\end{array}$ & \\
\hline $\begin{array}{l}\text { Compra de material } \\
\text { errado }\end{array}$ & $\begin{array}{l}\text { Diseño del instructivo para llenado del } \\
\text { formato, capacitación al personal que } \\
\text { diligenciarían el formato. }\end{array}$ & & X & & & & & $\begin{array}{l}\text { Mantener controles } \\
\text { aplicados }\end{array}$ & \multirow{9}{*}{$\begin{array}{l}\text { Diseño de un plan de } \\
\text { adquisición de } \\
\text { recursos de } \\
\text { información, } \\
\text { elaborado } \\
\text { conjuntamente con } \\
\text { vicerrectoría } \\
\text { académica y el } \\
\text { comité de selección } \\
\text { y adquisición }\end{array}$} \\
\hline $\begin{array}{l}\text { Compra de material } \\
\text { errado }\end{array}$ & $\begin{array}{l}\text { Envío de catálogo de material a } \\
\text { Docentes y directores de los diferentes } \\
\text { Departamentos y Programas para su } \\
\text { divulgación }\end{array}$ & & X & & & & & $\begin{array}{l}\text { Mantener controles } \\
\text { aplicados }\end{array}$ & \\
\hline $\begin{array}{l}\text { Demora, cambio o } \\
\text { rechazo de material } \\
\text { solicitado por parte del } \\
\text { comité de compras }\end{array}$ & $\begin{array}{l}\text { Cumplimiento de procedimientos } \\
\text { internos. Seguimiento realizado a la } \\
\text { aprobación del comité de compras. }\end{array}$ & & X & & & & & $\begin{array}{l}\text { Mantener controles } \\
\text { aplicados }\end{array}$ & \\
\hline $\begin{array}{l}\text { Rechazo de material } \\
\text { solicitado por parte del } \\
\text { comité de compras }\end{array}$ & $\begin{array}{l}\text { Se realiza argumentación del caso } \\
\text { previo a la discusión del material } \\
\text { (Soportes técnicos) }\end{array}$ & & $x$ & & & & & $\begin{array}{l}\text { Mantener controles } \\
\text { aplicados }\end{array}$ & \\
\hline $\begin{array}{l}\text { Compra de material } \\
\text { errado por error en la } \\
\text { solicitud }\end{array}$ & $\begin{array}{l}\text { Se realiza verificación del material } \\
\text { revisado en el área de circulación y en } \\
\text { Catalogación, comparando formato de } \\
\text { recepción contra orden de compra }\end{array}$ & & $X$ & $X$ & & & & $\begin{array}{l}\text { Mantener controles } \\
\text { aplicados }\end{array}$ & \\
\hline $\begin{array}{l}\text { Recepción de material } \\
\text { obsoleto o en mal } \\
\text { estado }\end{array}$ & $\begin{array}{l}\text { Inspección visual en el momento de } \\
\text { recepción. Material con signos de } \\
\text { deterioro debe ser preparado para ser } \\
\text { enviado al centro de reciclaje. }\end{array}$ & & $X$ & & & & $x$ & $\begin{array}{l}\text { Mantener controles } \\
\text { aplicados }\end{array}$ & \\
\hline $\begin{array}{l}\text { Oportunidad de } \\
\text { enriquecimiento de la } \\
\text { colección y mejora de } \\
\text { la Visibilidad } \\
\text { Institucional }\end{array}$ & $\begin{array}{l}\text { Establecer responsable de gestión con } \\
\text { entes externos. Seguimiento con } \\
\text { indicador asociado }\end{array}$ & $x$ & $x \mid x$ & & & & & $\begin{array}{l}\text { Implementar cambios } \\
\text { y realizar seguimiento } \\
\text { de cada caso. }\end{array}$ & \\
\hline $\begin{array}{l}\text { Contacto con nuevas } \\
\text { instituciones para } \\
\text { fortalecer las alianzas } \\
\text { institucionales }\end{array}$ & $\begin{array}{l}\text { Correo de contacto a instituciones } \\
\text { actuales. }\end{array}$ & $x$ & & & & & & $\begin{array}{l}\text { Establecer política } \\
\text { interna de revisión } \\
\text { anual de contactos. } \\
\text { Proponer intercambios } \\
\text { de otros materiales } \\
\text { propios. }\end{array}$ & \\
\hline $\begin{array}{l}\text { Recepción de material } \\
\text { obsoleto o en mal } \\
\text { estado }\end{array}$ & $\begin{array}{l}\text { Inspección visual en el momento de } \\
\text { recepción. Material con signos de } \\
\text { deterioro debe ser preparado para ser } \\
\text { enviado al centro de reciclaje. }\end{array}$ & & $X$ & & & & & $\begin{array}{l}\text { Mantener controles } \\
\text { aplicados }\end{array}$ & \\
\hline $\begin{array}{l}\text { Recepción de material } \\
\text { incompleto }\end{array}$ & $\begin{array}{l}\text { Verifica material contra remisión, orden } \\
\text { de compra y/o pedido. Llamar a } \\
\text { proveedores o instituciones } \\
\text { involucradas }\end{array}$ & & $x$ & & & & & $\begin{array}{l}\text { Check list con la } \\
\text { documentación que } \\
\text { debe ser entregada }\end{array}$ & $\begin{array}{l}\text { Contar con espacios } \\
\text { y recursos de } \\
\text { información } \\
\text { pertinentes, según } \\
\text { los grupos de interés }\end{array}$ \\
\hline
\end{tabular}


Tabla 4: continuación

\begin{tabular}{|c|c|c|c|c|c|c|c|c|}
\hline $\begin{array}{l}\text { Incorrecta } \\
\text { interpretación y } \\
\text { aplicación de las } \\
\text { normas de } \\
\text { catalogación y } \\
\text { clasificación } \\
\text { bibliográficas }\end{array}$ & $\begin{array}{l}\text { Implementación de Formato Validación } \\
\text { de Criterio para Catalogación de } \\
\text { material, como medida de control de } \\
\text { catalogación y clasificación de registros } \\
\text { bibliográficos. }\end{array}$ & $x$ & $x$ & & & & $\begin{array}{l}\text { Mantener controles } \\
\text { aplicados }\end{array}$ & \\
\hline $\begin{array}{l}\text { Truncamiento en la } \\
\text { colocación de los } \\
\text { códigos de barras en } \\
\text { el material } \\
\text { bibliográfico. }\end{array}$ & $\begin{array}{l}\text { Verificación en sistema de los códigos } \\
\text { de barra aplicados al material. } \\
\text { Seguimiento durante el proceso de } \\
\text { préstamo }\end{array}$ & $x$ & $x$ & & & & $\begin{array}{l}\text { Mantener controles } \\
\text { aplicados. Reporte a } \\
\text { catalogación en caso } \\
\text { de detectarse error de } \\
\text { lectura que imposibilite } \\
\text { el préstamo }\end{array}$ & \\
\hline $\begin{array}{l}\text { Error en la ubicación } \\
\text { del material } \\
\text { bibliográfico en } \\
\text { estantería. }\end{array}$ & $\begin{array}{l}\text { Verificación diaria aleatoria del material } \\
\text { por turno. Refuerzos internos de cómo } \\
\text { enganchar correctamente el material. }\end{array}$ & $x$ & $x$ & & & & $\begin{array}{l}\text { Mantener controles } \\
\text { aplicados. Realizar } \\
\text { anualmente arreglo de } \\
\text { colección junto con } \\
\text { inventario. }\end{array}$ & \\
\hline $\begin{array}{l}\text { Daño o pérdida del } \\
\text { material bibliográfico }\end{array}$ & $\begin{array}{l}\text { Limpieza y preservación del material } \\
\text { bibliográfico durante los procesos de } \\
\text { inventario. }\end{array}$ & $x$ & $x$ & & & & $\begin{array}{l}\text { Planificación de } \\
\text { campañas de cuidado } \\
\text { del material } \\
\text { Bibliográfico por parte } \\
\text { de los usuarios. } \\
\end{array}$ & \\
\hline $\begin{array}{l}\text { Afectación de la salud } \\
\text { por agentes biológicos } \\
\text { externos. }\end{array}$ & $\begin{array}{l}\text { Proceso de limpieza rutinario. Uso de } \\
\text { mascarillas y guantes para } \\
\text { manipulación del material durante los } \\
\text { procesos de ordenamiento. }\end{array}$ & & & & & $x$ & $\begin{array}{l}\text { Incluir en presupuesto } \\
\text { la compra de equipos } \\
\text { especializados. } \\
\text { Mantener los controles } \\
\text { actuales }\end{array}$ & \\
\hline $\begin{array}{l}\text { Datos errados en el } \\
\text { tipo de colección }\end{array}$ & $\begin{array}{l}\text { Protocolo escrito en formato Word, de } \\
\text { diligenciamiento del formato de } \\
\text { préstamo y revisión de material, en el } \\
\text { catálogo. } \\
\text { Capacitación del personal }\end{array}$ & $x$ & $x$ & & & & $\begin{array}{l}\text { Mantener controles } \\
\text { aplicados }\end{array}$ & \\
\hline $\begin{array}{l}\text { Envío de material } \\
\text { equivocado }\end{array}$ & $\begin{array}{l}\text { El encargado de atención recibe la } \\
\text { devolución por parte del usuario final y } \\
\text { gestiona el material correcto }\end{array}$ & $x$ & $x$ & & & & $\begin{array}{l}\text { Mantener controles } \\
\text { aplicados }\end{array}$ & \\
\hline $\begin{array}{l}\text { Falta de disponibilidad } \\
\text { de material para } \\
\text { prestación del servicio }\end{array}$ & $\begin{array}{l}\text { Revisión del material con editoriales } \\
\text { para actualizar la colección, } \\
\text { capacitación en recursos electrónicos }\end{array}$ & $x$ & $x$ & & & & $\begin{array}{l}\text { Mantener controles } \\
\text { aplicados }\end{array}$ & \\
\hline $\begin{array}{l}\text { Falla del fluido } \\
\text { eléctrico o del software }\end{array}$ & Formato NO LUZ & & & $x$ & & & $\begin{array}{l}\text { Mantener controles } \\
\text { aplicados }\end{array}$ & \\
\hline $\begin{array}{l}\text { Multa no justificada al } \\
\text { usuario }\end{array}$ & $\begin{array}{l}\text { Política de condonar multas si el } \\
\text { material aparece dentro de la colección } \\
\text { sin la respectiva descarga. }\end{array}$ & $x$ & $x$ & & & & $\begin{array}{l}\text { Mantener controles } \\
\text { aplicados }\end{array}$ & \\
\hline $\begin{array}{l}\text { Sobornos al personal } \\
\text { de circulación, } \\
\text { ofrecimiento de } \\
\text { dadivas o beneficios } \\
\text { económicos a cambio } \\
\text { de préstamos no } \\
\text { registrados u omisión } \\
\text { de multas }\end{array}$ & $\begin{array}{l}\text { Restricción de los permisos de acceso y } \\
\text { eliminación a los auxiliares }\end{array}$ & & & & $x$ & & $\begin{array}{l}\text { Refuerzo de las } \\
\text { normas internas al } \\
\text { personal (Cuando } \\
\text { aplique }\end{array}$ & \\
\hline \begin{tabular}{l|} 
Daño de formatos de \\
préstamo, agotamiento \\
del usuario durante la \\
solicitud del servicio
\end{tabular} & $\begin{array}{l}\text { Asesoría al usuario para minimizar error } \\
\text { en el diligenciamiento. }\end{array}$ & & & & & $x$ & $\begin{array}{l}\text { Rediseño de proceso } \\
\text { actual para migración } \\
\text { en línea (En curso con } \\
\text { Sistemas) }\end{array}$ & \\
\hline \begin{tabular}{l|} 
Riesgo de caída a \\
diferente nivel por \\
traslado del material \\
en brazo, en las \\
escaleras.
\end{tabular} & $\begin{array}{l}\text { Ubicación del personal de catalogación } \\
\text { en primer piso para evitar el traslado }\end{array}$ & $x$ & $x$ & & $x$ & $x$ & $\begin{array}{l}\text { Plan de redistribución } \\
\text { de espacios y } \\
\text { reubicación del } \\
\text { personal de } \\
\text { catalogación en primer } \\
\text { piso para evitar el } \\
\text { traslado }\end{array}$ & $\begin{array}{l}\text { Construir espacios } \\
\text { flexibles diseñados } \\
\text { para biblioteca, que } \\
\text { reconozcan estilos } \\
\text { de aprendizaje y } \\
\text { requerimientos de } \\
\text { los investigadores }\end{array}$ \\
\hline
\end{tabular}


Tabla 4: continuación

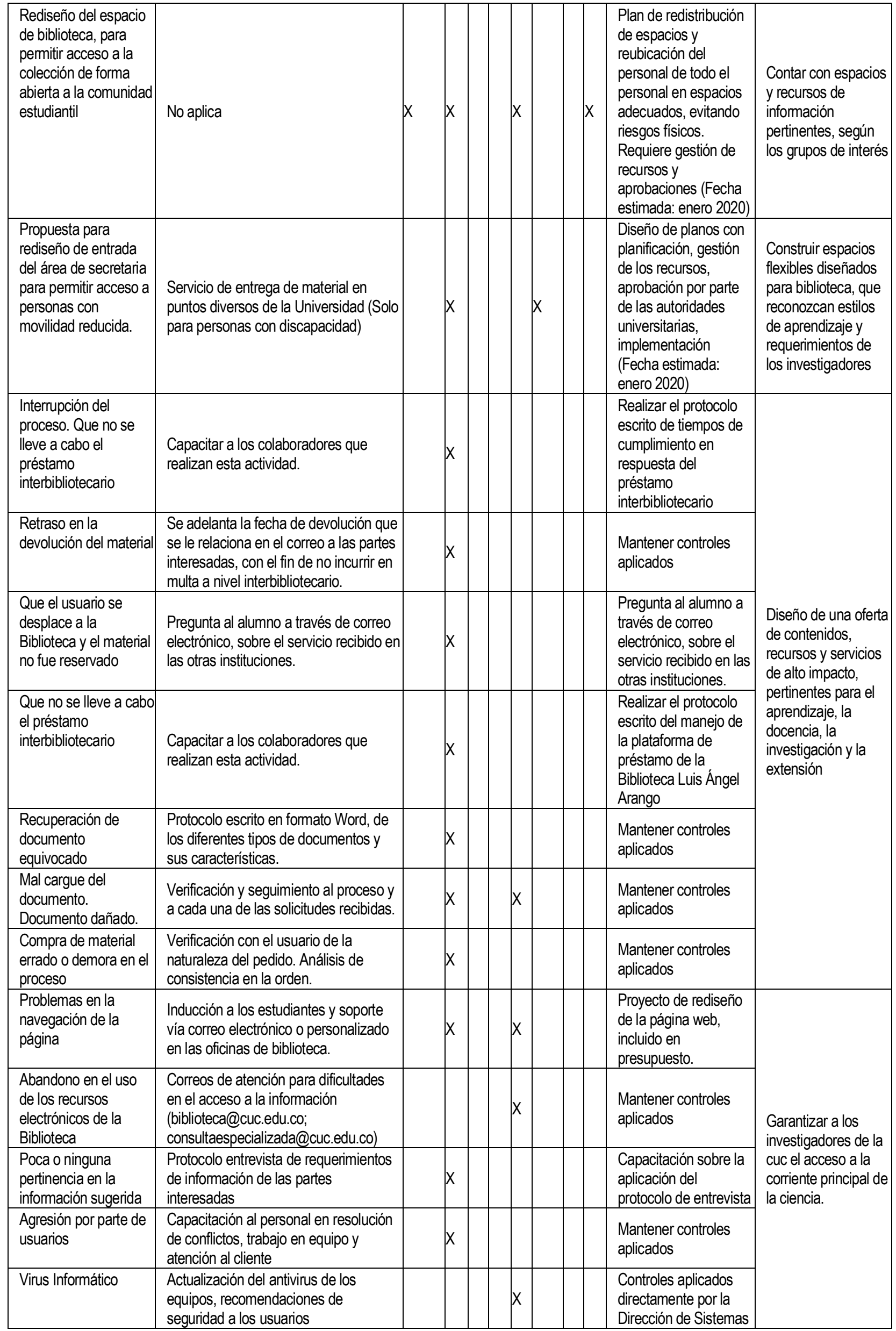




\section{DISCUSIÓN FINAL}

El Sistema de Gestión de la calidad, con el pasar del tiempo se ha transformado en una herramienta, de organización y certificación de los procesos a nivel mundial, la cual tiene como propósito planear, controlar, y mejorar los procesos de las organizaciones. En tal sentido, es importante señalar que la Universidad donde se realiza el estudio sobre la biblioteca universitaria posee Certificación ISO 9000, lo que representa que sus procesos y funciones se encuentran definidas y estandarizadas, en las diversas áreas, así como los indicadores o KPI de la institución, permitiendo alcanzar el objeto de la institución, como lo es la calidad de servicio.

El sistema de la gestión de la calidad, se encuentra implementado desde el año 2015, sin embargo, la norma está relacionada con el proceso de mejora continua, razón por la cual el objeto de la presente investigación, estuvo enmarcado en implementar el SGSI en los procesos de calidad de la Biblioteca de la Universidad, a través de la metodología MAGERIT, donde los resultados obtenidos permitieron establecer: Durante el proceso de implementación de la metodología, se lograron identificar los riesgos asociados a cada proceso, de acuerdo a la metodología el sistema de gestión de calidad, presenta ausencia en la identificación de amenazas y vulnerabilidades, por cada riesgo identificado.

Así como, el nivel de impacto debe ser evaluado, de acuerdo a las amenazas y vulnerabilidades, el SGC actual, refleja ausencia con relación a salvaguardas, por amenaza y vulnerabilidad detectada, lo cual hace que los controles para reducir o mitigar los riesgos sea muy general, para su tratamiento, los cálculos relacionados con los porcentajes de riesgos intrínseco y efectivo, demuestran que la presencia de salvaguardas y la evaluación de los impactos, afectan de manera positiva, permitiendo identificar el porcentaje de afectación en cada riesgo por proceso de calidad, lo cual permitió establecer la medida correctiva en cada caso, generando así el historial de acciones, finalmente la incorporación de los formatos propuestos, en los formularios se transformaría en una mejora, logrando así la implementación efectiva del SGSI para los procesos de calidad en la biblioteca seleccionada.

\section{CONCLUSIONES}

De acuerdo al trabajo presentado y a los resultados obtenidos, se pueden plantear las siguientes conclusiones principales:

En primera instancia se concluye que los sistemas de gestión de la seguridad de la información es uno de los puntos focales para el desarrollo de las instituciones educación superior; especialmente en el ámbito de la transferencia tecnológica y la investigación, donde la tecnología y la solidez de los sistemas informáticos frente a posibles amenazas es crucial para la integridad de la información depositada en dichos sistemas. Se concluye a su vez, que el nivel de impacto de un sistema de seguridad de la información debe ser evaluado con respecto a los actuales sistemas existentes en las instituciones; revisando sus vulnerabilidades y sus las amenazas; determinando de esta manera la presencia de los diversos riesgos en los procesos de calidad institucionales.

Del mismo modo, se concluye que la propuesta desarrollada se presenta como una alternativa de gestión de la seguridad de la información enfocada en las instituciones de educación superior; donde se espera que su aplicación en el campo permita demostrar la efectividad de la misma frente a las crecientes amenazas informáticas que se dan el día de hoy; especialmente frente al importante auge de las instituciones de educación superior en Colombia; las cuales lideran un proceso de desarrollo tecnológico en el panorama internacional.

\section{REFERENCIAS}

Akinola, S.A., Security of Library Resources and Crime Challenges in Nigerian University Libraries: A Look at the Situation in Joseph Ayo Babalola University, Ikeji Arakeji. Library Philosophy and Practice, ISSN: 1522-0222, 4080, 1-22 (2020)

Bauer, S., y Bernroider, E.W., From information security awareness to reasoned compliant action: analyzing information security policy compliance in a large banking organization, https://doi.org/10.1145/3130515.3130519, ACM SIGMIS Database: the DATABASE for Advances in Information Systems, 48(3), 44-68 (2017)

Bravo, M.J., Desarrollo de un Sistema de Gestión de Seguridad de la información para bibliotecas basado en una metodología mejorada para análisis de riesgos compatible con la norma ISO/IEC 27001: 2013, Tesis de maestría, Facultad de ingeniería de sistemas, Escuela Politécnica Nacional-Ecuador (2018)

Chang, K., Gao, Y., y Lee, S.C., The Effect of Data Theft on a Firm's Short-Term and Long-Term Market Value, http://dx.doi.org/10.3390/math8050808, Mathematics, 8(5), 1-21 (2020)

Chávez, N., Introducción a la investigación educativa, 2a Ed, Ars Gráfica Editores, 1-325, Maracaibo, Venezuela, (2007) 
Dirección General de Modernización Administrativa, Procedimientos e Impulso de la Administración Electrónica, MAGERIT versión 3: Metodología de Análisis y Gestión de Riesgos de los Sistemas de Información, Ministerio de Hacienda y Administraciones Públicas, Madrid, España (2012)

Donaldson, D.R., Certification information on trustworthy digital repository websites: A content analysis, http://dx.doi.org/10.1371/journal.pone.0242525, Plos one, 15(12) (2020)

Guamán, V.L., Evaluación de seguridad de la información aplicado al sistema de evaluación de docentes de la Universidad Técnica del Norte basado en la ISO 27002: 2017 con la metodología magerit V3, Tesis de maestría, Dpto. de Ingeniería en Ciencias Aplicadas, Universidad Técnica del Norte-Ecuador (2019)

Haghighat, M.H., y Li, J., Intrusion detection system using voting-based neural network, https://doi.org/10.26599/TST.2020.9010022, Tsinghua Science and Technology, 26(4), 484-495 (2021)

Hernández, H.G., Cardona, D.A., y Del Rio, J.L., Direccionamiento Estratégico: Proyección de la Innovación Tecnológica y Gestión Administrativa en las Pequeñas Empresas, http://dx.doi.org/10.4067/S0718-07642017000500003, Información Tecnológica, 28(5), 15-22 (2017)

ISO/IEC 27001:2013, Norma Técnica NTC-ISO/IEC Colombiana 27001, Bogotá, Colombia (2013)

Jamandilović, S., y Stojanović, M., Improving the security of the library information system by applying standard ISO 27001, Bosniaca: časopis Nacionalne i univerzitetske biblioteke Bosne i Hercegovine, ISSN: 1512-5033, 23(23), 95-98 (2018)

Khan, S.A., y Ayesha, G., Key features of information management systems (IMSs) for automation in university libraries: a view point of information professionals in Pakistan, https://doi.org/10.1108/LHT-04-2020-0087, Library Hi Tech (2021)

Lay, N., Márceles-Guerrero, V., y otros 9 autores, Uso de las herramientas de comunicación asincrónicas y sincrónicas en la banca privada del municipio Maracaibo (Venezuela), Revista Espacios, ISSN:0798-1015, 40(4), 11-21 (2019)

Liu, W., Huang, Q., Chen, X., y Li, H., Efficient functional encryption for inner product with simulation-based security, https://doi.org/10.1186/s42400-020-00067-1, Cybersecurity, 4(1), 1-13 (2021)

Qi, S., Lu, Y., y otros 3 autores, Cpds: Enabling Compressed and Private Data Sharing for Industrial Internet of Things Over Blockchain, https://doi.org/10.1109/TII.2020.2998166, IEEE Trans. Industrial Informatics, 17(4), 2376-2387 (2020)

Safa, N. S., Von Solms, R., y Furnell, S., Information security policy compliance model in organizations, https://doi.org/10.1016/j.cose.2015.10.006, Computers \& Security, 56, 70-82 (2016)

Sánchez-Otero, M., García-Guiliany, J., Steffens-Sanabria, E., y Hernández, H., Estrategias Pedagógicas en Procesos de Enseñanza y Aprendizaje en la Educación Superior incluyendo Tecnologías de la Información y las Comunicaciones, http://dx.doi.org/10.4067/S0718-07642019000300277, Información Tecnológica, 30(3), 277-286 (2019)

Susanto, H., y bin Muhaya, F., Multimedia information security architecture framework. In 5th International Conference on Future Information Technology, https://doi.org/10.1109/FUTURETECH.2010.5482696, IEEE, 1-5, Busan, South Korea (2010)

Tigănoaia, B., Some Aspects Regarding the Information Security Management System within Organizations-Adopting the ISO/IEC 27001: 2013 Standard, https://doi.org/10.24846/v24i2y201508, Studies in Informatics and Control, 24(2), 201-210 (2015)

Valencia, F.J., y Orozco-Alzate, M., Metodología para la implementación de un Sistema de Gestión de Seguridad de la Información basado en la familia de normas ISO/IEC 27000, https://doi.org/10.17013/risti.22.73-88, Revista Ibérica de Sistemas e Tecnologias de Informação, 22, 73-88 (2017)

Villarreal, S., García, J., Hernández, H., y Steffens, E., Competencias docentes y transformaciones en la educación en la era digital, http://dx.doi.org/10.4067/S0718-50062019000600003, Formación Universitaria, 12(6), 3-14 (2019)

Zhang, X., Corporate accounting information disclosure based on FPGA and neural network, https://doi.org/10.1016/j.micpro.2021.103973, Microprocessors and Microsystems, 81 (2021)

Zhao, Q., Chen, S., y otros 3 autores, Blockchain-based privacy-preserving remote data integrity checking scheme for loT information systems, https://doi.org/10.1016/j.ipm.2020.102355, Information Processing \& Management, 57(6), 102355 (2020) 